\title{
The Envelope of a Digital Curve Based on Dominant Points ${ }^{\star}$
}

\author{
David E. Singh ${ }^{1}$, María J. Martín ${ }^{2}$, and Francisco F. Rivera ${ }^{1}$ \\ 1 Univ. Santiago de Compostela, Dept. of Electronics and Computer Science, Spain \\ david@dec.usc.es, fran@dec.usc.es \\ 2 Univ. A. Coruña, Dept. of Electronics and Systems, Spain \\ mariam@udc.es
}

\begin{abstract}
In this work, we present an optimal solution to the following problem: given a Freeman chain-code curve with $n$ elements, and $m$ points of it, find the minimum envelope of the curve by a set of line segments. This segments are obtained modifying the coordinates of these $m$ points up to a distance $h$. The complexity of this algorithm is $O\left(n h+m h^{2}\right)$, and it needs a storage of $O(m h)$ data. In addition, we propose a greedy approximation algorithm that provides good results with lower complexity $O(n h)$ in the worst case, and memory requirements $O(h)$. A pre-processing with $O(m n)$ is also needed for both algorithms. Some experimental results are shown.
\end{abstract}

\section{Introduction}

Computing minimal enclosures for geometric curves is a fundamental geometric optimization problem. This problem arises in applications in which one wishes to find an approximation to a curve that is simpler in the sense of having a smaller number of elements. The main difference respect of the polygonization of digital curves is that we impose the enclosure restriction. The problem of computing minimum convex polygonal enclosures of a given number of sides for a convex polygon was efficiently solved by Aggarwal and Park [1]. They studied monotone matrix-searching techniques for this optimization problem.

Related work has been done on finding a minimal enclosing triangle of a convex polygon [2], a minimal enclosing $\mathrm{k}$-gon [3], a minimal enclosing equiangular k-gon [4] and minimum polygon enclosures with specified angles [5].

Several heuristics are available for "approximating" a contour by a set of line segments [6],[7] according to various criteria. All these methods are computationally expensive (usually between $\mathrm{O}\left(n^{2}\right)$ and $\mathrm{O}\left(n^{3}\right)$ ). Although most of these works try to produce good results, few attempts have been made so far to actually yield strictly optimal solutions under well-defined optimization criteria.

In this work we present an optimal solution to the following problem:

Given a sequence of $n$ integer-coordinate points and assuming that the curve is represented using the Freeman chain-code, we describe a digital curve $C$ as:

\footnotetext{
* This work was supported by the Xunta de Galicia grant PGIDT99PXI20602B
} 
$C=\left\{p_{j}=\left(x_{j}, y_{j}\right), j=1, \ldots n\right\}$

Where $p_{j+1}$ is a neighbour of $p_{j}$. The purpose of this work is finding another sequence of $m$ points that we will call $E=\left\{d_{j}, j=1, \ldots m\right\}$ where $m<n$. The digital curve related to $E$ will be the one conformed by joining these points by means of segments. This curve is defined as a set of linear functions: $F=\left\{f_{k}, k=\right.$ $1, \ldots m-1\}$ so that $f_{k}$ is the segment that joins $d_{k}$ and $d_{k+1}$. The sequence of points $E$ has to fulfill the following two conditions:

1. Its associated closed digital curve $F$ is envelope of $C$.

2. Defining $S(C)$ and $S(F)$ as the number of inner points to $C$ and to $F$ respectively. The goal is to get is the minimum value for $|S(C)-S(F)|$ that is referred to as error area.

As a starting point, we define $E$ as a subset of $m$ representative points of $C$. This subset should offer the maximum possible information on the shape of $C$, so that its digital associated curve be as similar as possible to $C$. It can be proved [8] that the most characteristic points in $C$ will be those that show the biggest curvature, which are called dominant points. However, the digital curve associated to $E$ may intersect $C$, not fulfilling the first demanded condition to be an envelope. Therefore, we propose an algorithm that carries out slight modifications on the position of the points in $E$ so that their associate digital curve can verify both conditions.

In this work two different techniques are suggested to obtain the envelope. One of them estimates the best solution in terms of minimizing the error area; since that starting from a initial $E$ set, the coordinates of their elements are modified until an associated curve is obtained with the smallest error area. The second is a greedy heuristic solution that although doesn't secure the best result, finds a close estimate with less computational and memory requirements.

The digital curves that we have used show the following restriction: the values of $x_{j}$ are monotonous regarding $j$, that is to say, $0 \leq x_{j+1}-x_{j} \leq 1$. In case that the digital curve doesn't fulfill this restriction, it can be broken down in tracts that do fulfill it and each can be analyzed independently. In order to obtain the envelope, and to maintain this property of the digital curves, we have imposed a limitation, that is the only coordinate that could be modified in the position of the dominant points is its $y$ coordinate.

For the calculation of the dominant points of $C$ we have used the Kankanhalli algorithm [9]. This means an improvement on the Teh-Chin algorithm [10] in which computational work is strongly reduced. Also, it maintains two important characteristics of the original algorithm: the fact that the entry parameters are not required, and the determination of the dominant points based on the local curvature. We have modified the algorithm allowing the selection of the dominant points based on their importance. This will allow us to select a smaller number of points knowing that these they will be the most significant in the curve. Anyway, the algorithms could be applied to another set of points. An alternative proposed by [11] use naive lines to obtain this points.

Once obtained the first initial set $E$ with an arbitrary number of dominant points of the curve, the following step, common to both proposals, is to obtain 
information on the characteristics of the $C$ curve among serial elements of $E$. The following section describes how to obtain that information.

\section{Obtaining Information of the Curve}

This preprocessing operates on the tract of $C$ between two consecutive dominant points $\left\{d_{i}, d_{i+1}\right\} \in E$ that we will call $d_{l}$ and $d_{r}$ respectively. The analysis of each tract is independent of the rest of them. If we define as $d_{l}^{k}$ the point $\left(x_{l}, y_{l}+k\right)$, the purpose of this step is to obtain, for each $d_{l}^{k}$, the points of tangency $t_{l}^{k} \epsilon C$. These are defined as the points belonging to the intersection of $C$ with the straight line that goes through element $d_{l}^{k}$ and that is tangent to $C$ in the segment $\left\{d_{l}, d_{r}\right\}$. A representation of these points can be seen in Figure 1. For a given $d_{l}^{k}$, and its corresponding $t_{l}^{k}$, by means of an extrapolation of the tangent straight line, the position of the associated element $d_{r}^{k^{\prime}}$ can be obtained. Notice that the minimum value of $k$ is zero, and the maximum is the one in which the corresponding point $d_{r}^{k^{\prime}}$ belongs to $C$, or equivalently $k^{\prime}=0$.

It is useful some structure like the one shown on Table 1 to store all the relevant information on the process. The values that are shown are those corresponding to the situation given in Figure 1. This structure has a column for each possible value of $k$.

In order to obtain this table, the entry data are the digital curve $C$ evaluated in the segment $\left\{d_{l}, d_{r}\right\}$ and, inside that interval, the element with the maximum value of $y$ coordinate: $p_{\max }=\left(x_{\max }, y_{\max }\right)$. This point can be obtained without additional complexity during the calculation of the dominant points.

Table 1. Data structure associated to a tract of $C$

\begin{tabular}{|c|c|c|c|c|}
\hline Levell & Level1 & Level2 & Level3 & Level4 \\
\hline$d_{l}^{0}$ & $d_{l}^{1}$ & $d_{l}^{2}$ & $d_{l}^{3}$ & $d_{l}^{4}$ \\
\hline$t_{l}^{0}$ & $t_{l}^{1}$ & $t_{l}^{2}$ & $t_{l}^{3}$ & $t_{l}^{4}$ \\
\hline$d_{r}^{4}$ & $d_{r}^{3}$ & $d_{r}^{2}$ & $d_{r}^{1}$ & $d_{r}^{0}$ \\
\hline
\end{tabular}

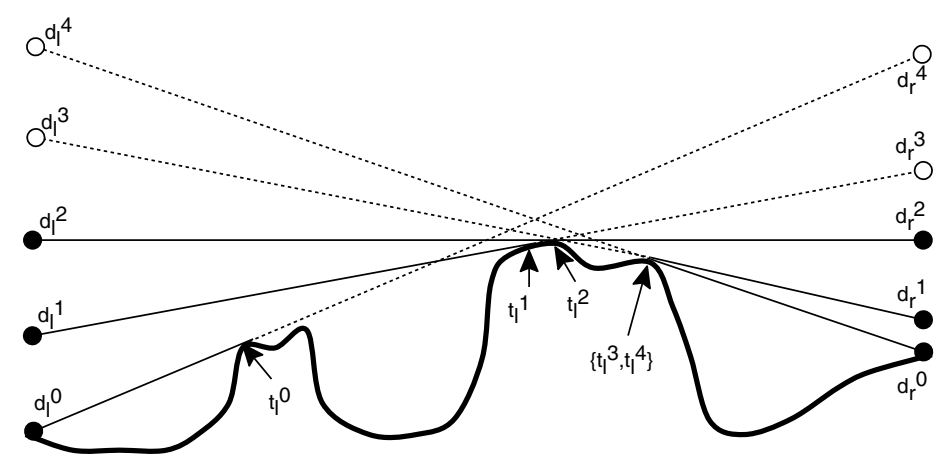

Fig. 1. Representative points of a tract of $C$ 
The process is divided in two equivalent problems: obtain the $t_{l}^{k}$ in the interval $\left[x_{l}, x_{\max }\right]$ for different $d_{l}^{k}$ values, and $t_{r}^{k^{\prime}}$ in the interval $\left[x_{\max }, x_{r}\right]$ for different $d_{r}^{k^{\prime}}$ values. The values of $k$ and $k^{\prime}$ allowed will be those that verify respectively, the following conditions: $k \epsilon\left[y_{l}, y_{\max }\right]$ and $k^{\prime} \epsilon\left[y_{r}, y_{\max }\right]$. We should point out that, although the problem has been broken into two halves, it is only necessary one access to the points of $C$, since these two halves are disjointed. If the curve has more than a maximum value, then the first and the last of them have to be considered, being irrelevant in the calculations the intermediate points of $C$.

An outline of the used algorithm is shown below. Since the resolution of both problems is equivalent, the shown algorithm corresponds to the resolution of the tract of $C$ among $d_{l}$ and $p_{\max }$ that we call $C^{\prime}$. The other part of the tract is solved in the same way going over curve $C$ from $d_{r}$ to $p_{\max }$.

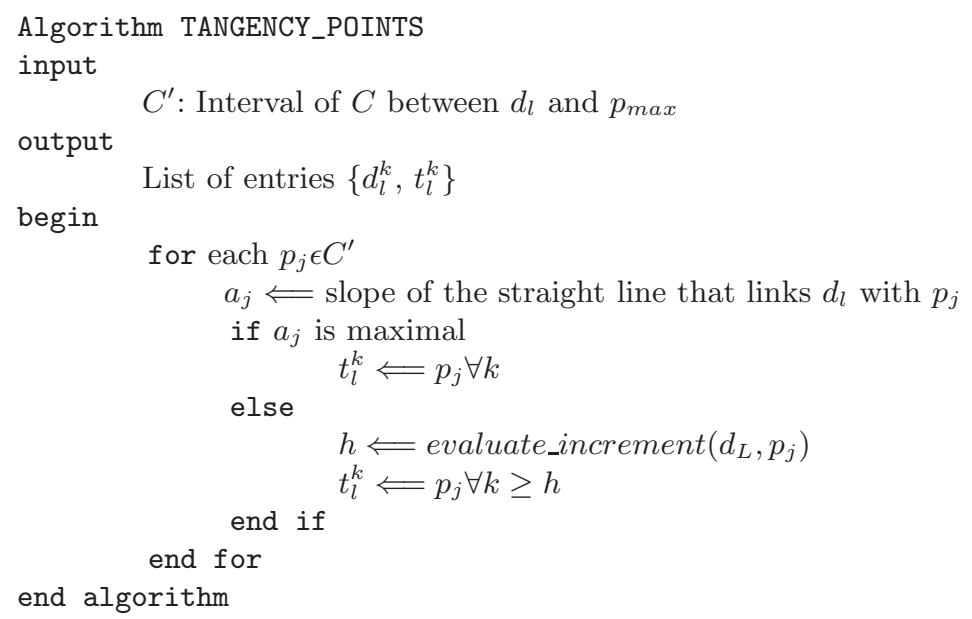

This algorithm is based on two properties[12]:

1. Given a point $p_{j} \epsilon C$ and the slope $a_{j}$ of the straight line that joins it with $d_{l}$. If the value of this slope is higher than the one obtained for the previous elements with a lower abscissa, then none of these previous elements is tangential. Therefore, the considered point is a tangential point for all $d_{l}^{k}$.

2. Given a point $p_{j} \epsilon C$ that doesn't verify the previous condition, but with a $y$ coordinate higher than the $y$ coordinate of the element $p_{j^{\prime}}$ that has given the maximum slope. Then, this point is tangential to all the $d_{l}^{k}$ that have a value for $k$ equal or higher than the one given for the Expression 1.

$$
h=\frac{a_{j^{\prime}}-a_{j}}{x_{j}-x_{j^{\prime}}}\left(x_{j^{\prime}}-x_{l}\right)\left(x_{j}-x_{l}\right)
$$

Through the outermost loop in the algorithm, the points of the digital curve inside the considered interval are considered, and we check whether they verify property 1 . To carry out this, it is only necessary to store the maximum value of the slope of the processed points. Among all the points that do not verify it, it is only necessary to consider those with a value in their coordinate $y$ higher than the one of the point which has given the maximum slope. For these points, equation 1 is used to obtain the value for $k$ for which they become tangential. 
There is a question that is not mentioned in the algorithm due to lack of space, that is checking that the rest of the entries $t_{l}^{k}$ with $k>h$ are not associated to another tangential point. If it is noticed that any $t_{l}^{k}$ is assigned to another element $p_{j^{\prime}}$ different from the one of the maximum slope (another tangential point exists) and it is necessary to evaluate again expression 1 between $p_{j}$ and the new element.

Carrying out a similar process on the interval $\left[x_{\max }, x_{r}\right]$ one can obtain the position of the tangency points for the different $d_{l}^{k}$. Combining both results and extrapolating the values, all the $d_{l}^{k}$ and $d_{r}^{k^{\prime}}$ positions higher than $p_{\max }$ can be obtained as shown in Figure 1. In this figure, the dashed lines link the points that are extrapolated.

\section{Obtaining the Optimal Envelope}

The problem lies in figuring out the sequence of $d_{i}^{k}$ points whose associate digital curve $F$ is a minimum envelope. This method makes use of an exhaustive search among all the possible envelopes to the curve. For each $d_{i}^{k}$ element belonging to the tract, the neighbour of the previous tract is searched so that when joined, the associate curve has a minimum error area. An example can be seen in Figure 2, in which two serial tracts of $C$ are represented that span between points $\left\{d_{1}, d_{2}, d_{3}\right\}$. Point $d_{3}^{0}$ can be joined with $d_{2}^{2}$, so that the segment is tangent to $C$. However, it can also be joined to $d_{2}^{3}$ or the $d_{2}^{4}$ satisfying the condition of being a envelope, and although locally it is not the optimum, it can be part of the global optimum.

The number of combinations for joining serial dominant points would grow exponentially with the number of tracts unless the condition of minimizing the error area was demanded. Due to this, the search space in our algorithm decreases considerably and it is possible to enunciate the following property: for a certain dominant point $d_{i}^{k}$ there is at least one neighbour $d_{i-1}^{k^{\prime}}$ with which it can form a minimum envelope [12].

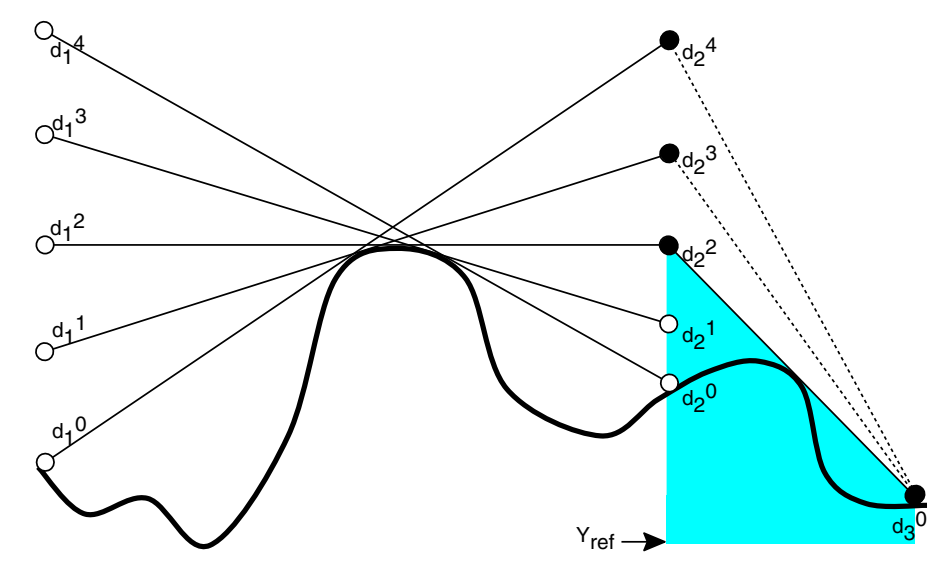

Fig. 2. Possible links between $d_{3}^{0}$ and $d_{2}$ 
Each tract is limited by two elements $\left\{d_{i}, d_{i+1}\right\} \in E$ and has associated a table with all the possible tangency points. This table is obtained with the method described in the previous section. Each level contains the following fields: $\left\{d_{i}^{k}, t_{i}^{k}, d_{i+1}^{k^{\prime}}\right.$, area, link $\}$. The field area stores the error area accumulated in that tract, and link indicates the element of the structure immediately previous to the one the current level is connected to.

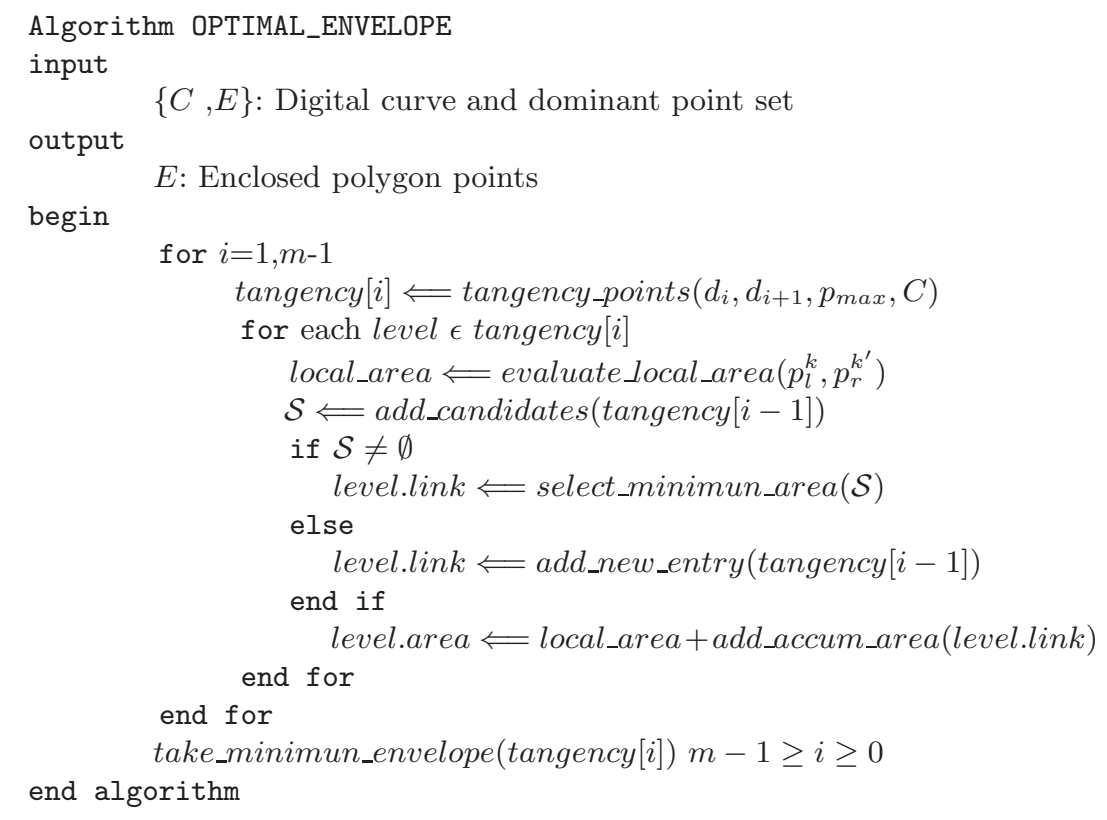

The OE algorithm is shown above. It successively processes the different tracts of $C$. For each one, the algorithm obtains the table with the possible tangency points, storing them in the structure called tangency. Next, it goes over all the levels of the generated table. For each level of the table the function evaluate_local_area() is executed. This function obtains the area of the surface embraced by the tangent segment to $C$ regarding a coordinate of reference $y_{\text {ref }}$. The value of this area is proportional to the error area, so that the entry that obtains the lowest value will represent the tangential tract that minimizes the area of error of the interval. In Figure 2, the tangent segment to $C$ associated to point $d_{3}^{0}$ is the one that joins it to $d_{2}^{2}$. The local area appears shadowed in the figure for that situation. Notice that this calculation is independent of the element to which is linked.

For each $d_{i}^{k}$ it is necessary to calculate the set $\mathcal{S}$ of levels of the previous table with which it can be joined. This is carried out by function add_candidates(). For example, in Figure 2, the group associated to $d_{3}^{0}$ would be $\mathcal{S}=\left\{d_{2}^{2}, d_{2}^{3}, d_{2}^{4}\right\}$. For each one of the possible candidates the accumulated area is evaluated. For the union $d_{3}^{0}-d_{2}^{2}$ the area will be the one that this last point has accumulated plus the local one to $d_{3}^{0}$. However, for the union $d_{3}^{0}-d_{2}^{4}$ the area of the triangle formed by points $d_{3}^{0}-d_{2}^{2}-d_{2}^{4}$ has to be added to the above-mentioned one. 


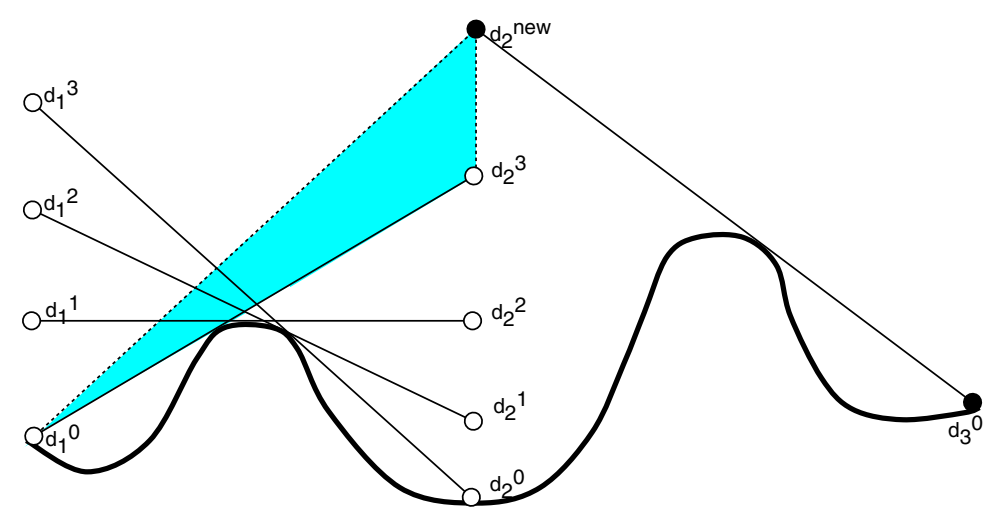

Fig. 3. New level in $d_{2}$ due to $d_{3}^{0}$

Of all the possibilities of union, the one that obtains the smallest accumulated area is selected since it minimizes the area of global error of that level. Applying this procedure iteratively, it is sure that, when the possible values for $d_{i}^{k}$ are analyzed, the accumulated areas of all the $d_{j}$ with $j<i$ will be the minimum.

A particular situation that is necessary to consider is when there is no element of the previous table that can be joined to the entry under consideration. This is the case, in Figure 3, of point $d_{3}^{0}$. In this situation, and in order to maintain the coherence of the information, by means of add_new_entry () a new entry is created in the previous table that contains the new value of $d_{i-1}^{k}$ with which it can be joined. The rest of the fields of this new entry are the same that of the entry $d_{i-1}^{k-1}$ immediately lower. In the figure, the entry $d_{2}^{\text {new }}$ associated to the point $d_{3}^{0}$ would be created. The only exception is the accumulated error area, since it is necessary to bear in mind the increment in area in a similar way to how we did in the previous section. Again, in the figure it would be necessary to add to the accumulated area associated to point $d_{2}^{3}$, the area of triangle $d_{1}^{0}-d_{2}^{3}-d_{2}^{\text {new }}$. An important property is that the link of this new entry is the same that the one of the immediately lower entry. This is because the area difference accumulated among both entries (the triangle) is independent of the element to which is linked. As the union of the previous entry ( $d_{2}^{3}$ in the figure) is the one with the smallest accumulated area of all the possibilities, that will also be the case for the new entry.

When all the intervals have been processed, only the entry of the last tract with the smallest accumulated area is taken. Next, we go back over the previous tracts and by means of the field link, we obtain the sequence of points $E$ whose associated digital curve has the smallest error area. 


\section{An Heuristic Method of Obtaining the Envelope}

The method described in the previous section makes it certain obtaining a minimum error area. However it has several inconveniences: the structures called tangency of each tract must be stored, all the entries of each one of them should be analyzed and it is necessary to go over them twice, one to obtain all the possible solutions and the other one, in the opposite direction, to extract the best one. In this section we show a greedy heuristic method with much less computational work, less demanding in terms of memory and that provides a solution close to the best one.

The HE algorithm is shown below. The different intervals of $C$ are processed consecutively calculating for each of them the tangency points. The difference regarding the previous method is that now a local reduction is intended in the error area. For each interval, only the central dominant point $d_{i}$, and the two immediate neighbours $d_{i-1}$ y $d_{i+1}$, are considered. Next, an increase in the coordinate $y_{i}$ of $d_{i}$ is conducted, this might imply a decrease of the $y$ coordinate of their two neighbours as it is shown in Figure 4.

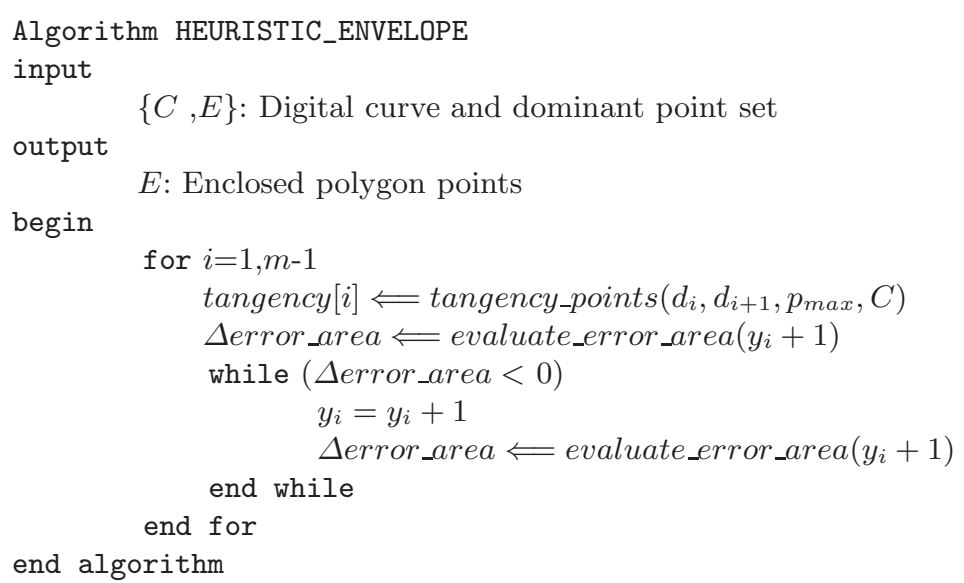

The increment experienced in the error area derives from the expression $\Delta$ error_area $=(A 2+A 3)-(A 0+A 1+A 4)$, where the first term takes into account the gained error area and the second the lost one. For each modification in $d_{i}$ starting from the tables tangency $[i]$ and tangency $[i-1]$ the value of the new positions of $d_{i-1}$ and $d_{i+1}$ are obtained. Once known, we check whether a net reduction in the error area has taken place and we move on to evaluate the following increase in $y_{i}$. It can be easily shown that the previous relationship can be rendered in a function that is dependent only on the coordinates $x$ of the points[12]. Expression (2) is verified if only if $\Delta$ error_area $<0$.

$\left(x_{\beta}-x_{\alpha}\right)\left(x_{2}-x_{\alpha}\right)\left(x_{\beta}-x_{2}\right)<\left(x_{\alpha}-x_{1}\right)\left(x_{\alpha}-x_{0}\right)\left(x_{\beta}-x_{2}\right)-\left(x_{3}-x_{\beta}\right)^{2}\left(x_{2}-x_{\alpha}\right)$

Where $x_{\alpha}$ and $x_{\beta}$ correspond to the abscissas of the point of intersection of the segment with the initial position and the one that has the final position. 
This expression allows us to determine, in a quick way, if there is a reduction in the error area or not. This process verifies the following property: when for some value $y_{i}$ the previous inequality can no longer be verified, for the remaining higher than $y_{i}$ values it will not be verified either [12].

Out of this property we can conclude that starting from the last $d_{i}$ that verifies the inequality there will not be any other one that produces a smaller error area. This way the algorithm of local minimizing of the area should only evaluate the inequality for point $y_{i}+1$. If this is verified, then $y_{i}$ is incremented. This process will be repeated until the inequality no longer is satisfied.

\section{Results}

The computational complexity of the $\mathrm{OE}$ algorithm is $\mathrm{O}\left(n h+m h^{2}\right)$. The number of entries that are needed to store the information according to Table 1 is $\mathrm{O}(m h)$. By contrast, in the case of the HE algorithm, the complexity is $\mathrm{O}(n h)$, and the amount of memory needed to store the information is $\mathrm{O}(h)$. Several rugged curves were used as benchmark to prove and compare both algorithms. We use a curve of 1666 points to illustrate the results, and similar behaviours were obtained on other curves. A section of this curve with different number of points and its envelope is shown in Figure 5, notice the irregularity of its shape. A preprocessing with $O(m n)$ is also needed for both algorithms.

Figure 6(a) shows the execution time consumed by both methods when they are run on a SUN Enterprise 250 computer. Notice that the reduction in runtime is important; in some cases is even less that half. Figure 6(b) shows the maximum amount of memory needed to store the tangency tables. The great improvement in this issue is clear. Finally, figures 6(c) and 6(d) show the comparison in terms of number of error pixels between both methods. The difference is important when $m$ is low ( $40 \%$ with 3 points) but it fastly lows to $1 \%$ or less.

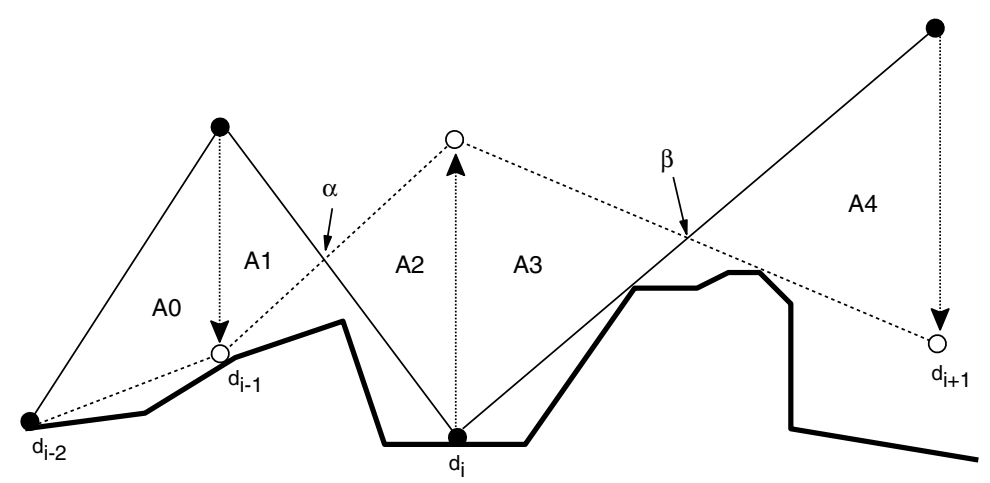

Fig. 4. Effect of increasing $y_{i}$ 


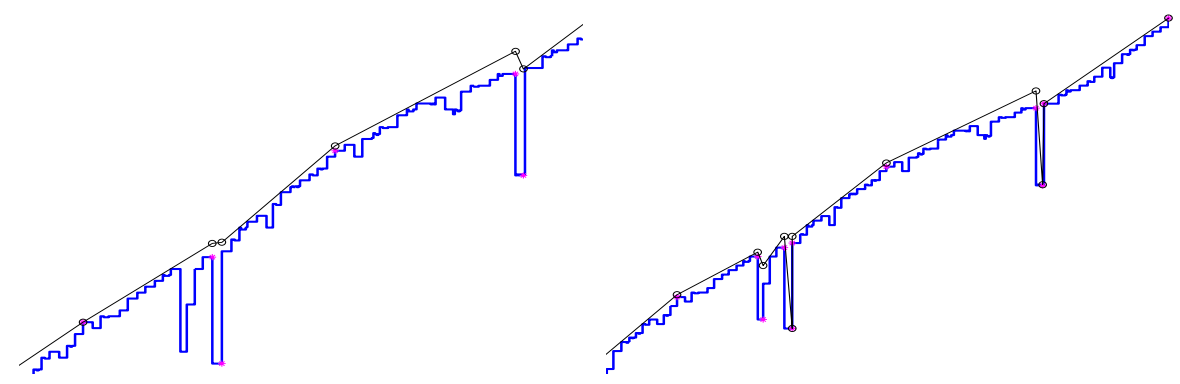

Fig. 5. Curve examples

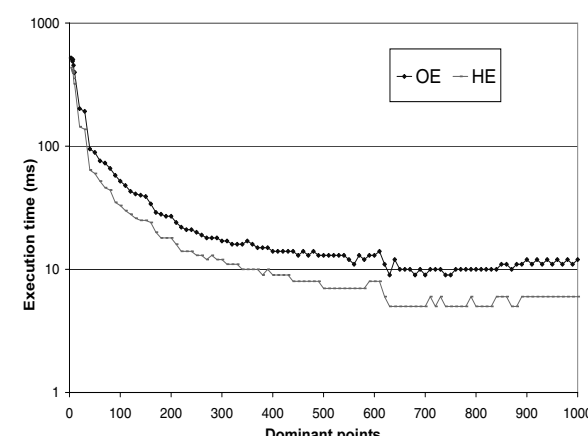

(a) Execution time

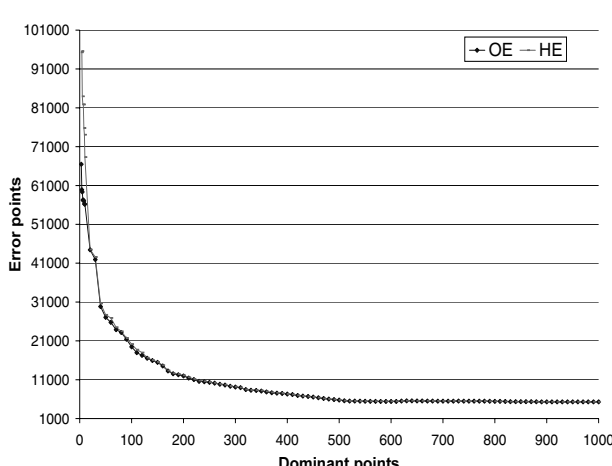

(c) Error area

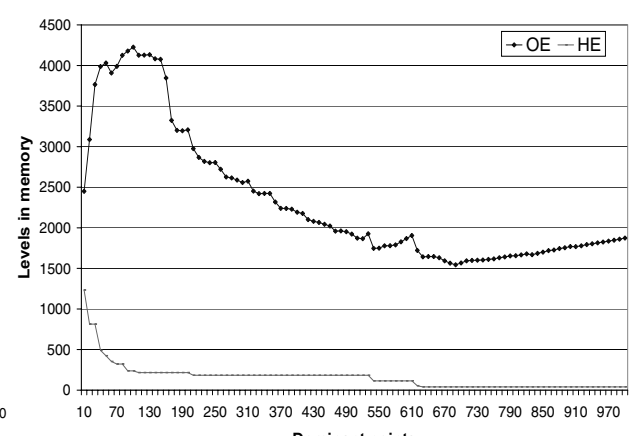

(b) Memory requirements

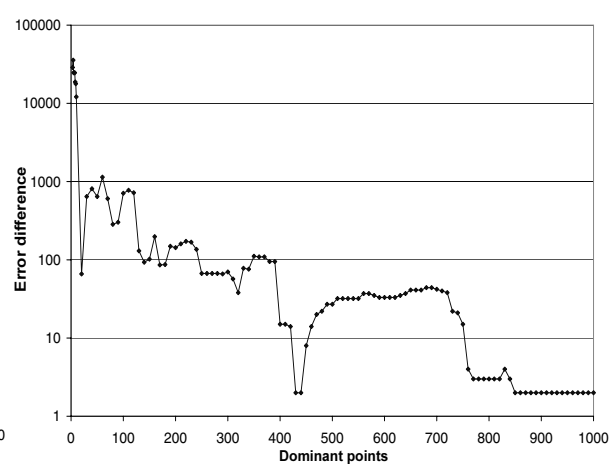

(d) Difference of error area

Fig. 6. Efficiency of both approaches 


\section{References}

1. A. Aggarwal, J. Park: Notes on Searching in Multidimensional Monotone Arrays. Proc. 29th IEEE Symp. on Found. of Comp. Sci. (1988), 497-512 453

2. J. O'Rourke, A. Aggarwal, S. Maddial, M. Baldwin: An optimal algorithm for finding enclosing triangles. J. Algorithms, 7 (1986) 258-269 453

3. A. Aggarwal, J. Chang, C. Yap: Minimum Area Circumscribing Polygons. Visual Computer, 1 (1985), 112-117 453

4. A. DePano, A. Aggarwal: Finding restricted $k$-envelopes for convex polygons. Proc. 22nd Allerton Conf. on Comm. Control and Computing, (1984) 81-90 453

5. David M. Mount, Ruth Silverman: Minimum enclosures with specified angles. Technical Report, CS-TR-3219 (1994) 453

6. J. G. Dunham: Optimum Uniform Piecewise Linear Approximation of Planar Curves. IEEE Trans. Pattern Anal. and Machine Intelligence, V. 8-1, (1986) 67-75 453

7. Paul L. Rosin: Techniques for Assessing polygonal approximations of curves. IEEE Trans. on Pattern Anal. and Machine Intelligence, Vol 19, No 6 (1997) 659-666 453

8. F. Attneave: Some informational aspects of visual perception. Psychol. Review. vol 61, no 3, (1954) 183-193 454

9. Mohan S. Kankanhalli: An adaptive dominant point detection algorithm for digital curves. Pattern Recognition Letters. 14 (1993) 385-390 454

10. Cho-Huak Teh, Roland T. Chin: On the detection of dominant points. IEEE Trans. on Pattern Anal. and Machine Intelligence. Vol 11. No 8 (1989) 859-872 454

11. Isabelle Debled-Rennesson, Jean-Pierre Reveillès: A linear algorithm for segmentation of digital curves. Int. J. of Pattern Recog. and Artificial Intelligence. 9-6 (1995) 454

12. David E. Singh, María J. Martín, Francisco F. Rivera: Propiedades geométricas en la obtención de la envolvente a una curva digital. Tech. report. (1999) 456, 457, 460,461 